\title{
Multiparty Micropayments for Ad Hoc Networks
}

\author{
Hitesh Tewari and Donal O'Mahony \\ NTRG, Dept of Computer Science \\ Trinity College \\ Dublin - 2, Ireland
}

\begin{abstract}
The majority of ad hoc networks and their associated applications have been designed with closed user groups in mind. In such scenarios all the nodes in the network usually belong to a single authority and are configured to cooperate in the relaying of packets within the network. In recent years however, ad hoc networks have also found their way into everyday networking environments, where mobile devices may be under the administrative control of individual users. These users may not necessarily be motivated to provide services for free to others in the network. A typical situation could be where a node may wish to relay packets through a number of intermediate nodes in the ad hoc network, to access services in the fixed network. In this paper we present a lightweight payment scheme based on hash chains, which allows a node to pay others who relay packets on its behalf in real-time. Also due to the dynamic nature of an ad hoc network, the topology of the network can change unpredictably. The design of the payment scheme is flexible enough to be able to cope with such route changes, without the need to contact a trusted third party such as a bank or broker to pay the nodes in the new path.
\end{abstract}

\section{INTRODUCTION}

Ad hoc networks have become the topic of increased interest in recent years. An ad hoc network is an infrastructureless mobile network, which consists of a group of nodes that communicate with each other using multihop wireless links. Each node acts as a router to forward packets for other nodes within the network. Most of the current work has focused on closed user groups, where nodes in the path between the source and destination cooperate in the relaying of packets. In closed environments such as military or emergency services networks, all the nodes in the network belong to a single authority and are motivated to work with each other to achieve a common goal.

However the research community envisages a greater role for ad hoc networks in everyday life, such as extending the reach of the fixed network to the users within an ad hoc network. In such networks the individual nodes may have affiliations with different network operators or could be under the administrative control of individual users. These users may not necessarily wish to cooperate in the relaying of packets on behalf of other users in the network. One of the major concerns of such users will be to preserve the limited battery life of their mobile devices, and the relaying of packets directly impacts on this. In addition, one can imagine ad hoc networks as an alternative for cellular access networks in the future that will be used by end users to access services in the fixed network. Thus we must have mechanisms in place to compensate the nodes involved in the relaying process and for any value-added services that they provide. However in ad hoc networks we do not have the luxury of long-lived trust relationships between nodes in the network, as is the case in the wired Internet. Also it is not always possible to contact a trusted third part (TTP) to verify for example user identities or payment tokens that may be presented to a node. In the latter case, the cost of contacting a TTP to verify payment tokens may outweigh the actual benefits gained.

Previous work in this area has been done within the framework of the Terminodes project [1]. They have proposed a virtual currency called nuglets [2] that attempts to stimulate cooperation within the network by rewarding nodes for service provision. The authors indicate that there could be "selfish" nodes in the network that will use services provided by other nodes, but modify their own behavior such that they do not cooperate in providing free services to others in the network. Nodes in a Terminodes network are required to pay for services from other nodes using nuglets. In addition the authors feel that payment using nuglets on a per packet basis will deter users from overloading the network.

Since the only way to transmit data on the network is by paying other nodes using nuglets, it is in the interest of users to keep their nodes in an active state and accumulate nuglets by relaying packets for others. When transmitting a packet, a node attaches sufficient nuglets such that each intermediate relay node will acquire some of the nuglets to cover its forwarding costs. To prevent nodes from acquiring more than their fair share, the designers make use of secure hardware modules in each node. The secure hardware module maintains a nuglet counter and modifies its contents according to the number of nuglets received or spent by a node. To prevent forgery of nuglets contained within the packet purse during transmission, neighboring nodes use asymmetric key cryptography to negotiate symmetric keys to encrypt the packet contents.

In our opinion there are a number of drawbacks to the Terminodes approach. There is total dependence on the secure hardware module to protect nuglets in the system. If the module's security is compromised then a user can mint nuglets at his discretion and disrupt the cooperative nature of the system. Also there is no mechanism to determine the total cost of transporting a packet from a source to a destination. If the source node fails to attach sufficient nuglets with a packet, then an intermediate node prior to reaching its destination will drop the packet.

In this paper we outline a payment scheme that enables a node to join an existing ad hoc network, and allows it to pay each node that relays packets on its behalf in real-time. In 
section 2 we give an overview of micropayment technology and the use of hash chain trees as an efficient means for storing hash values and distributing micropayment tokens. Based on our understanding of current work in the area we present a number of protocol goals for an ideal payment system for ad hoc networks in section 3. In section 4 we present our multiparty payment scheme for ad hoc networks and discuss its various merits and drawbacks. Finally in section 5 we make some concluding remarks.

\section{MICROPAYMENT TECHNOLOGY}

Numerous methods for electronically paying for goods or services across a network have been proposed [3, 4, 5]. In [6] the authors provide a detailed description of some of the more important of electronic payment solutions and give an overview of payments methods for mobile networks. These systems are usually electronic equivalents of physical payment methods such as cash, checks or credit cards. Each of these macropayment instruments have a minimum transaction overhead, as they require an on-line connection to a bank or a trusted third party to verify the authenticity of the payment token. In addition most systems make use of computationally expensive cryptographic operations such as public key cryptography. These two factors combined make the use of macropayment systems prohibitive for payments of a few cents. In contrast micropayments are a family of payment systems which have been designed to allow repeated small valued payments e.g. one-tenth of a cent in a single transaction. Micropayment research has concentrated on repeated payments at a single vendor. Many of the schemes make use of one-way hash functions [7] to generate a chain of hash values. A one-way function is one where it is easy to compute $y=f(x)$ but computationally expensive to reverse the transaction. Hash functions such as MD5/SHA are more computationally efficient than symmetric key algorithms such as AES or asymmetric key algorithms such as RSA and allow for fast generation and verification of payment tokens.

\section{A. Micropayments Using Hash Chains}

A user generates a hash chain of length $\mathrm{N}$ by applying a hash function $\mathrm{N}$ times to a random value $\mathrm{P}_{\mathrm{N}}$ the root of the hash chain, to obtain a final hash $\mathrm{P}_{0}$ the anchor of the hash chain. The user commits to the chain by digitally signing the anchor with his private key. For each payment, the user releases the pre-image of the last hash value. For example for the first payment the user releases the hash value $P_{1}$. The receiver can apply the same hash function to the value $P_{1}$ to obtain the anchor $\mathrm{P}_{0}$. Since the hash function is one-way only the user could have generated the hash value.

Fig. 1 shows the overall payment process where a user generates a hash chain of length $\mathrm{N}$. The user creates a commitment by digitally signing the anchor of the chain $\mathrm{P}_{0}$, the length of the chain, the value of each hash and the vendor at which he wishes to spend the chain. Prior to payment the user forwards the commitment to the vendor, who can verify its authenticity off-line. For each micropayment the user releases the next payment hash in the chain. The vendor can redeem the hashes at the broker with whom the user has an account at a later date, by presenting the highest payment hash along with the signed commitment.

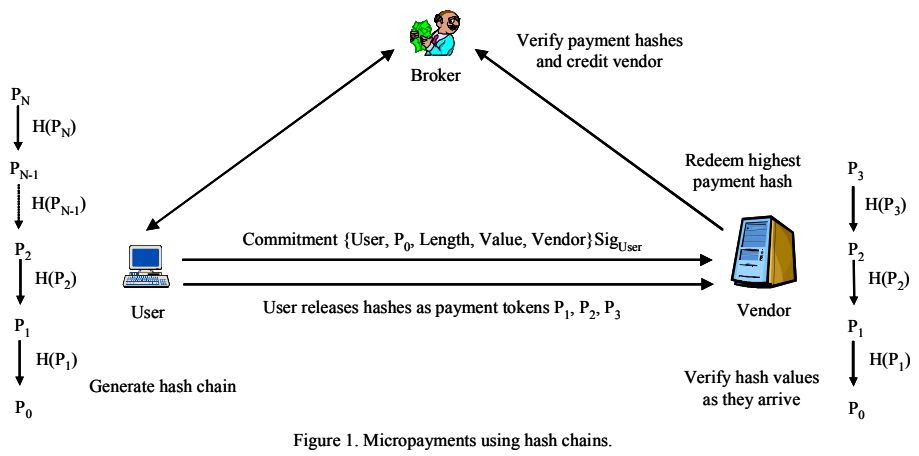

\section{B. UOBT Hash Chain Trees}

The unbalanced one-way binary tree (UOBT) scheme is an efficient hash chain scheme, where the root of each chain is derived from another hash chain [8]. The scheme is ideal when a large number of hashes are needed and the device has limited storage capabilities. Only the tree root value has to be stored on the device to be able to reconstruct the entire UOBT. Fig. 2 shows an example UOBT where $\mathrm{P}_{40}$ is the tree root.

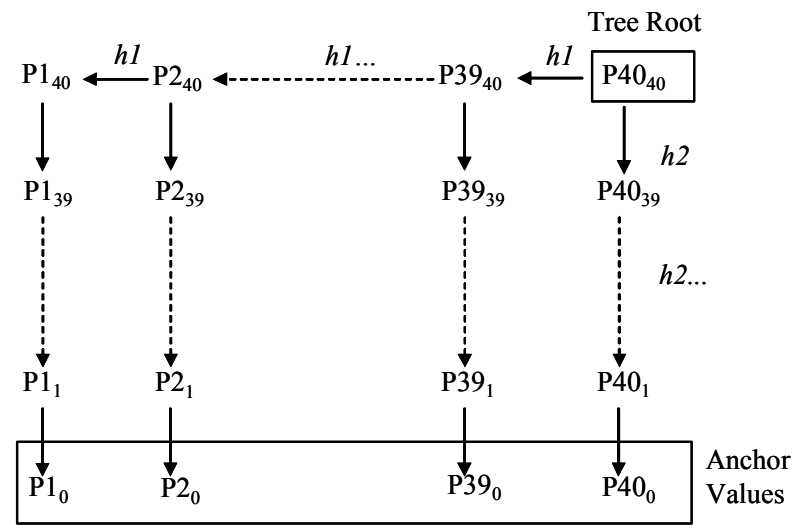

Figure 2. Unbalanced one-way binary tree.

We repeatedly apply the hash function $h 1$ to this value to obtain the backbone hash chain $\left(\mathrm{P}_{40} 0_{40}, \ldots \mathrm{P} 1_{40}\right)$. Each of these hash values is used as the secret root value for deriving the individual sub-chains by applying the hash function $h 2$. For example, the value $\mathrm{P} 2_{40}$ is the root of the $\mathrm{P} 2$ chain, which consists of the values $\mathrm{P} 2_{40}, \mathrm{P} 2_{39}$ and so on until the anchor of that chain $\mathrm{P} 2{ }_{0}$ is reached. The result is a UOBT with a backbone chain length of 40, with each sub-chain also consisting of 40 hash values and an overall hash chain consisting of 1600 hash values. The signed commitment consists of a hash of the concatenation of the anchors of each of the sub-chains $\left(\mathrm{P} 1_{0}, \mathrm{P} 2_{0}, \ldots \mathrm{P} 39_{0}, \mathrm{P} 40_{0}\right)$ signed with the private key of the user or broker.

On small devices with limited storage such as a PDA, it may not be possible to store all the hash values of a long hash chain. If a UOBT is used with a backbone chain length equal to the length of each sub-chain, it can be shown that the average computational overhead to compute the next hash is $n^{1 / 2}-1$. 
Where $n$ is the number of values in the UOBT, and $n^{1 / 2}$ is the square root of $n$. A 100x100 UOBT requires 99 hashes on average to compute a hash value. There is an initial overhead in transporting the set of anchor values to the broker for the commitment to be signed. However once the initial exchange has taken place, the number of cryptographic operations performed during the communications session are greatly reduced.

\section{SYSTEM OVERVIEW}

We envisage that in the future, ad hoc networks will complement existing mobile and fixed communications networks. A majority of the time nodes within an ad hoc network may communicate with each other. However as in current mobile systems, users in an ad hoc network may also wish to access services in the fixed network. For example a user might want to make voice calls to other mobile and PSTN users or access data services on the Internet. Nodes that are in close proximity to a fixed network can extend the reach of the fixed network to other nodes within a mobile ad hoc network. Consider the scenario illustrated in Fig. 3. Upon arrival in a new city a user finds a route through an ad hoc network to a node that has the capability to relay packets into the fixed network using existing ad hoc routing protocols. Each of the nodes in the path from the user to the fixed network must be actively involved in relaying the packets towards the destination.

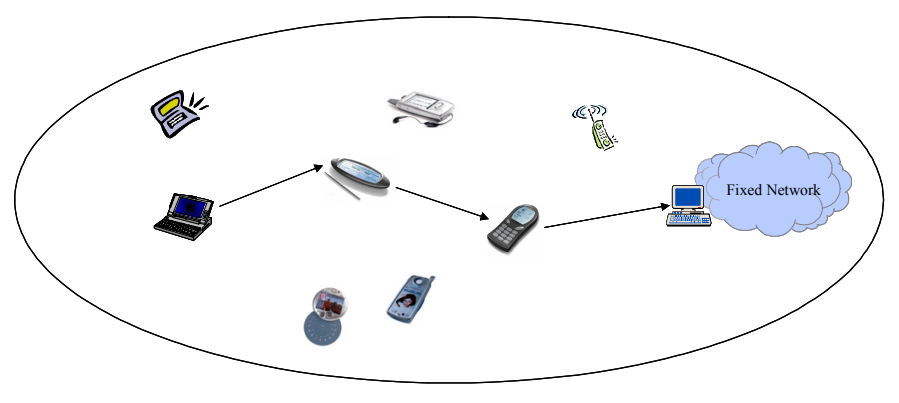

Figure 3. Ad hoc network

We propose a protocol employing micropayment technology, which allows each of the nodes involved in the relaying of the packets to be paid as they provide the service. In mobile and fixed networks the entities within the core network remain static. This allows a user to negotiate a payment contract to pay nodes [9] along the route to the destination. The payment contract can be bound to the identities of each of the nodes in the path and can be certified by a trusted third party. However such long-lived contracts are not feasible in ad hoc networks. Due to the dynamic nature of an ad hoc network, the route from the source to the destination may change over a period of time or even during a call. We require a more lightweight and flexible scheme that allows us to pay all nodes in the path to a given destination without the requirement to contact a TTP or a bank to issue a new payment contract.

\section{A. Protocol Goals}

The protocol has been designed to address the issue of payment for forwarding of packets by nodes in an ad hoc network. From our analysis of existing work in this area, we feel that any payment scheme employed in an ad hoc network must have the following goals:

- Off-line verification of payment tokens. Intermediate relay nodes should not be required to have an on-line connection to a bank or a trusted third party to verify payment.

- Flexibility in choosing routes. A node should be able to independently choose the most optimal route to its destination and pay all nodes along the path to forward packets on its behalf. If there is a change in the route towards the destination, then the source should not have to contact a third party to construct a new payment contract to pay the nodes in the new path.

- Lightweight cryptographic procedures. Intermediate nodes must be able to quickly verify the payment information carried within a packet. Use of heavyweight cryptographic algorithms may result in delays in packet forwarding by intermediate nodes.

- Minimize fraud in the system. The effort required to steal value from the system should be far greater than the rewards gained. Post-fact detection should identify the culprits, who can subsequently be disqualified from the system.

\section{PAYMENT FOR PACKET FORWARDING}

\section{A. Broker Commitment}

A user buys prepaid tokens through their terminal from a broker. A user must first establish an accounting relationship with a broker whose main purpose is to aggregate micropayments between entities. The broker supplies each account holder with a smart card, which is loaded with a public key pair that is bound to the user's identity by means of a digital certificate. The card also contains the broker's publickey certificate. The smart card is a secure and trusted tamper resistant device that acts on behalf of the broker. Prior to transmitting any packets on the network a user must purchase payment chains from the broker. A macropayment scheme such as a credit card or electronic cash can be used to make the purchase. Fig. 4 shows the payment chain purchase protocol. The user instructs the smart card module to generate for example a 40x40 UOBT. To obtain a broker commitment, the user forwards a signed message that consists of the set of anchors along with the length of the sub-chains, the value of a single hash and the macropayment details to the broker all encrypted with the brokers public key. The tree root of the UOBT and the associated secret root values never leave the secure hardware module on the user's device.

The broker generates a set of $\mathrm{N}$ secret endorsement values, one for each anchor value that was sent by the user. A broker endorsement consists of an anchor value $\mathrm{PX}_{0}$, the corresponding endorsement value (a random number), the length of the hash chain, the value of a hash in the chain, the identity of the user that purchased the chain and the expiry date of the chain. All of the above fields are signed with the private key of the broker. The endorsement allows a recipient to 


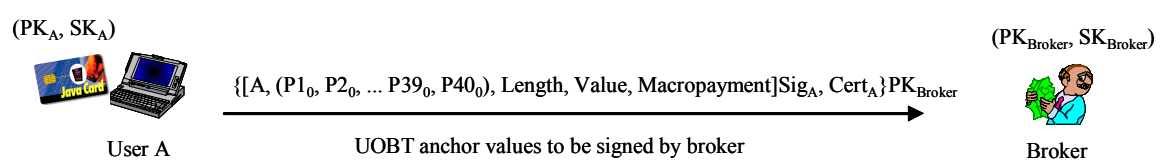

Broker generates secret endorsement values $\{\mathrm{E} 1, \mathrm{E} 2, \ldots \mathrm{E} 39, \mathrm{E} 40\}$
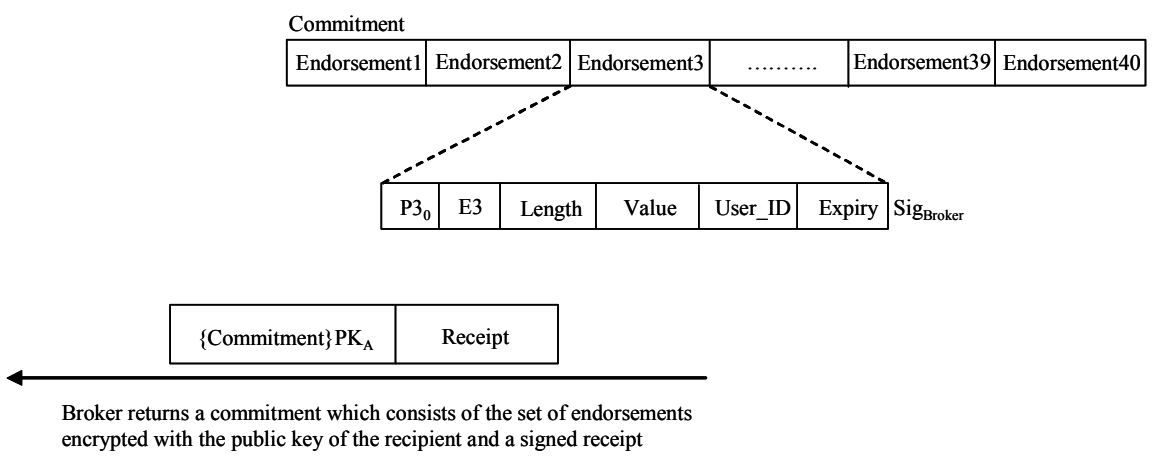

Figure 4. Purchase of payment chains and broker commitment.

redeem the highest hash value in the corresponding sub-chain of the UOBT at the broker. The commitment (set of broker endorsements) is encrypted with the public key of the user. The commitment can only be decrypted within the user's smart card hardware module. The broker also forwards a signed receipt to the user, which allows him to verify that the broker has committed to the sub-chains of the UOBT.

Receipt $=\left\{\mathrm{A},\left(\mathrm{P} 1_{0}, \mathrm{P} 2_{0}, \ldots \mathrm{P} 39_{0}, \mathrm{P} 40_{0}\right)\right.$, Length, Value, Expiry $\} \operatorname{Sig}_{\text {Broker }}$

The size of each broker endorsement is 40 bytes assuming that the lengths of the anchor and the corresponding endorsement value are 16 bytes each and that each of the other fields is assigned 2 bytes. The broker's signature with a 1024 bit key amounts to 128 bytes. For a 40x40 UOBT the total size of the broker commitment amounts to $(40 * 168) \sim 7$ Kbytes of data. For a UOBT of 100x100 the broker commitment would be $\sim 17 \mathrm{Kbytes}$. These values are within the storage capabilities of current high-end and next generation smart card devices.

The smart card, which acts on behalf of the broker, will not allow the user access to the endorsements. They will be only released in encrypted form from the user's smart card device to a recipient's smart card device prior to payment for packet forwarding. However, smart card devices are tamper resistant and not tamper proof. So even though we rely on these devices to ensure that a user cannot use a payment chain multiple times, we are not able to guarantee this. A determined user could break the tamper resistance of the smart card module and gain access to the broker signed endorsements. We limit the exposure of our system from such attacks by associating an expiry date with each chain, after which other users in the system will not accept the chain as a valid payment instrument.

\section{B. Charge Assembly and Endorsement Distribution}

The process of setting up a call to a remote destination requires that the initiating node have knowledge of the total costs involved in forwarding a packet through the ad hoc network. Each node in the path to the destination must indicate its charge for packet forwarding. We assume that the source node has knowledge of the routes through the network by making use of an existing reactive or proactive routing protocol. It may then subsequently query nodes along one or more of these routes to obtain the total charge for packet forwarding through the routers along that path. Fig. 5 shows the overall process of assembling the charging information. In this case we assume that node $\mathrm{A}$ has selected a route to the destination node $\mathrm{X}$ along the path that is served by nodes $\mathrm{B}, \mathrm{N}$ and $\mathrm{R}$. We assume that the source node can verify that it has obtained a valid route to the destination from the underlying routing protocol and that there are no loops in the route, which may have been formed by nodes colluding to defraud others in the network.

Node A requests the underlying routing protocol to find a route to the destination node $\mathrm{X}$ in the fixed network by sending it a charge request message. The nodes in the ad hoc network that are in the relay path to the destination attach a service offering which can have a number of different security levels. At a very minimum, a service offering could consist of a plaintext message with the number of hash tokens that a node requires to forward packets to the next hop. To prevent intermediate nodes from modifying the charge details in their favor, we can get each node to digitally sign the charge-related data with their private key as shown in Fig 5. An intermediate node will append one or more certificates, so that the source is able to verify the digital signature on the charge details. Finally to prevent replay attacks, a node could add a nonce to the message before signing it. This information is returned in a charge reply message back to node $\mathrm{A}$.

In this case nodes $\mathrm{B}$ and $\mathrm{N}$ specify a charge one hash token per packet, while the gateway node $\mathrm{R}$ specifies a charge two hash tokens per packet. Once the source has found the cheapest or most optimum route to the destination it distributes the broker signed endorsements to each of the relay nodes in the path. The endorsements are transferred from the secure hardware module in the source node to each of the recipients. Since the endorsements are secret quantities, they are encrypted with public key of each recipient which is obtained from the public-key certificate, that was forwarded with the charge reply 


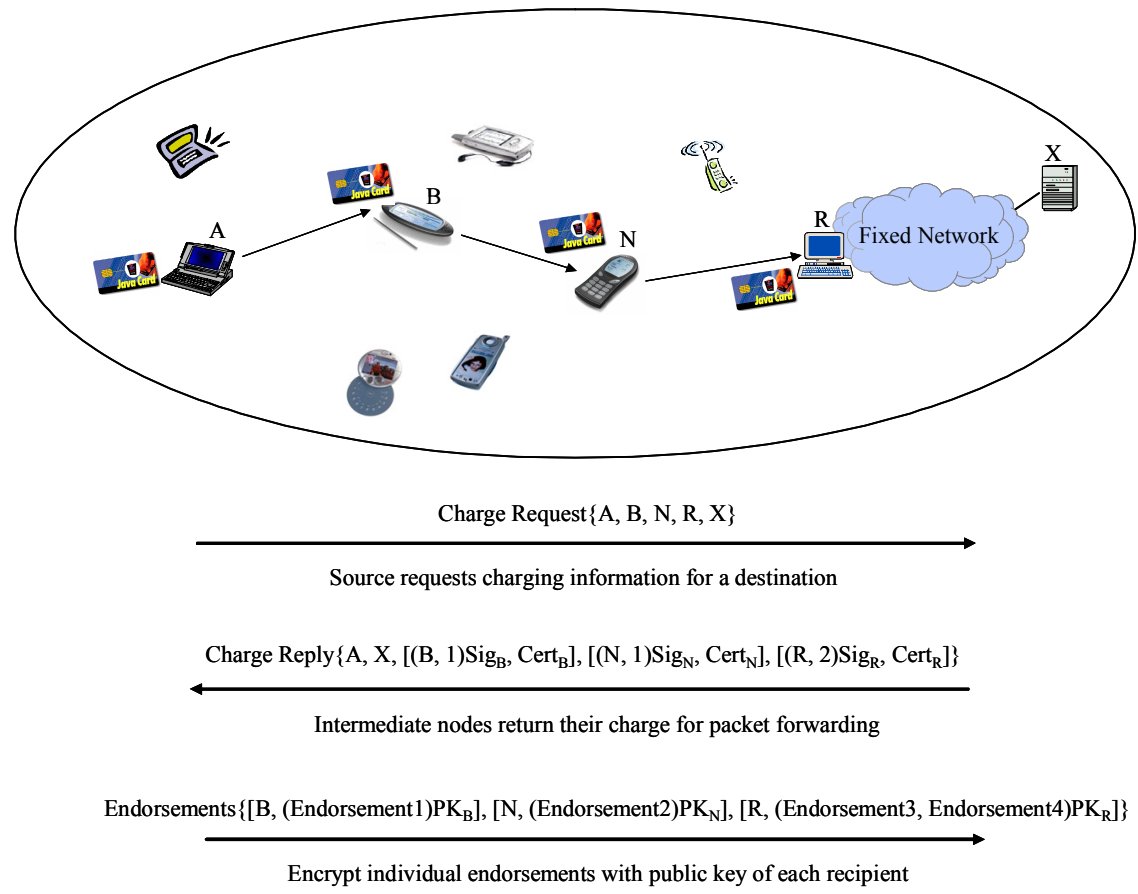

Figure 5. Endorsement distribution.

message. In Fig. 5 we encrypt Endorsement1 with the public key of node $\mathrm{B}$. This allows the source node to spend the first sub-chain $\left(\mathrm{P}_{0}, \ldots \mathrm{P} 1_{40}\right)$ of the UOBT with node B. Similarly we encrypt Endorsement 2 with the public key of node N. However to node $\mathrm{R}$ we send two endorsements namely Endorsement 3 and Enodorsemen4. This is necessary as we will use up twice as many hash tokens at node $\mathrm{R}$, in order for it to forward packets into the fixed network. The smart card module in each recipient node will verify the contents of the endorsements and the digital signature of the broker on the same.

Each of the nodes along the path to the destination in the ad hoc network is now ready to accept payment tokens for packet forwarding. Since the initial signaling messages are crucial to the workings of the protocol, we can assume that we will make use of a reliable transport protocol such as TCP to transport the same. This will ensure that signaling messages can still be delivered, even if packets are dropped due to congestion in the network. Alternatively if we are restricted to using an unreliable transport protocol such as UDP, then we may transmit the endorsements along with the first three data packets. This will ensure that all intermediate routers will have the required endorsement values to accept payment for packet forwarding. Also we assume that signaling or control messages will be routed through the system free of charge prior to any data transfer. Finally we note that for very small amounts of data transfer, the signaling overhead associated with distributing the payment parameters could exceed the user data.

\section{Making Payments}

For each packet or sequence of packets node A wishes to transmit into the fixed network, it attaches a single hash token from the sub-chains $\mathrm{P} 1_{0}, \mathrm{P} 2_{0}$ and two hash tokens from the subchain $\mathrm{P} 3_{0}$. Note that to pay a node multiple hash tokens we do not have to attach multiple hash values. Instead we can just attach the correct hash value further up the chain. For example the first packet contains the hash values $\mathrm{P} 1_{1}, \mathrm{P} 2_{1}$ and $\mathrm{P} 3_{2}$. Node $\mathrm{B}$ applies the corresponding hash function to $\mathrm{P} 1_{1}$ to obtain the anchor of the chain $\mathrm{P} 1_{0}$. It is able to verify that this is the value that was contained within the broker-signed endorsement that was given to it by node $\mathrm{A}$. Similarly node $\mathrm{N}$ is also able to quickly verify the authenticity of the hash token $\mathrm{P} 2{ }_{1}$. Node R can verify the hash tokens by applying the hash function twice to the value $\mathrm{P} 3_{2}$ to obtain the anchor of the chain $\mathrm{P} 3_{0}$. Fig. 6 depicts the overall process of payment using hash tokens.

Note that we do not need to protect the hash tokens using additional cryptographic procedures. Even though intermediate nodes have access to the hash values they will not be able to redeem them, as they require the corresponding broker signed endorsement. Under normal operation of the smart card device, the endorsements will remain within the secure hardware module of the smart card and not be released in the clear. Fig. 6 depicts the situation where the call has terminated after we have spent 30 hash tokens from the chains P1 and P2 at nodes $\mathrm{B}$ and $\mathrm{N}$ respectively. At node $\mathrm{R}$ the chain $\mathrm{P} 3$ has been exhausted and 20 hash values from the chain P4 have been used. The storage requirements at an intermediate node are 184 bytes for each chain that it is willing to accept. This figure comprises of 168 bytes for the broker signed endorsement and 16 bytes for the highest hash value received by the node.

\section{Change in Route - New Path}

Changes in the topology of the ad hoc network will result in a change in the route towards the fixed network or other nodes within the ad hoc network. However we are still required to pay each node along the new path to the destination. The design of our payment system is flexible enough that we are able to cope 

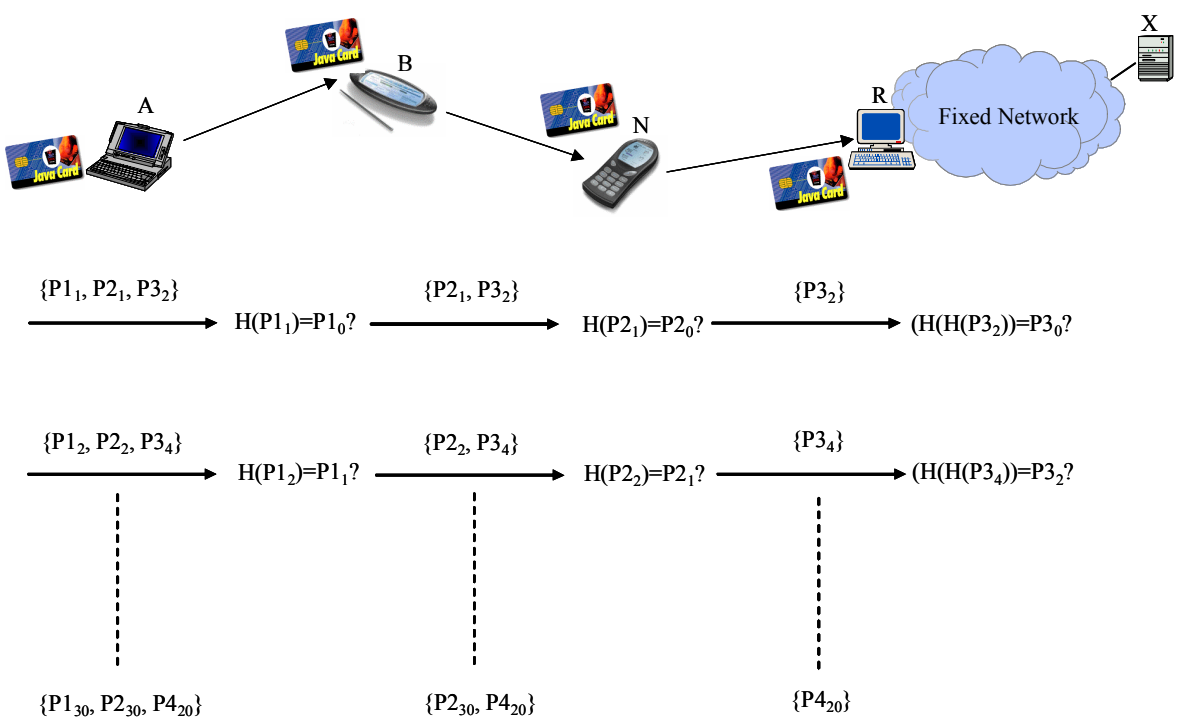

Figure 6. Releasing hash tokens for payment.

with this situation, without the need to contact a trusted third party or broker. Since the broker has committed to our UOBT that consists of multiple chains, we can immediately start paying each of the nodes in the new path. Fig. 7 depicts a situation where the new path to the fixed network from node A is via nodes $\mathrm{L}, \mathrm{N}$ and $\mathrm{R}$. As before each node indicates its charge for packet forwarding in the charge reply message. We note that the only new node in the path is node $\mathrm{L}$ and that we still have unspent chains with nodes $\mathrm{N}$ and $\mathrm{R}$. Instead of distributing a new set of endorsements to all nodes at the start of a new call, we only distribute endorsements to the new nodes in the path.

In the discussion above we distribute a single endorsement to node $\mathrm{L}$ and continue using the old chains at the other nodes in the path. At nodes $\mathrm{N}$ and $\mathrm{R}$ we can make use of chains $\mathrm{P} 2$ and P4 for which we had previously distributed endorsements. During the last call we used 30 hash values in chain P2, which means that there are still 10 unspent hashes left in that chain. Similarly we had 20 hash values left over in the chain P4. So in this case we forward just a single endorsement (Endorsement5) to node $\mathrm{L}$ encrypted with its public key. Along with the first packet we attach the hash tokens $\mathrm{P} 5_{2}, \mathrm{P} 2_{31}$ and $\mathrm{P} 4_{22}$. Node $\mathrm{L}$ performs two hash functions on the value $\mathrm{P}_{2}$ to obtain the anchor of the chain P5, which it can verify from the signed broker endorsement. Node $\mathrm{N}$ performs a single hash operation to obtain the value $\mathrm{P} 2_{30}$, which it has stored from the last call. Similarly node R can perform two hash operations on the value $\mathrm{P}_{22}$ to obtain the highest hash value $\mathrm{P} 4_{20}$ that it has stored from the last call. If we wish to continue the call after we have transmitted 10 packets from node $\mathrm{A}$, we must distribute a new set of endorsements to all the nodes in the relay path.

Our solution tries to maximize the number of hash values used in each sub-chain of the UOBT. However we only made partial use of the chain P5 at node L before having to distribute new endorsements to all the nodes in the path to continue the call. An alternative method could be used whereby we could make use of a chain from a shorter UOBT. A user could purchase multiple UOBTs of different lengths in advance from a broker. At node $\mathrm{L}$ we could then make use a sub-chain from a $20 \times 20$ UOBT or two sub-chains from a $10 \times 10$ UOBT. This would result in no wastage of hash tokens in those chains. This requires additional work on behalf of the source node to correctly identify new nodes in the relay path and keep track of unspent hash chains.

In most cases there will be a bi-directional exchange of packets. For example when a node requests a Web page from a remote server in the fixed network, it will receive data packets in response to that request. We need to ensure that the nodes in relay path will forward any response packets back to the source node. Assuming that there are symmetric radio links between neighboring nodes, there are a number of ways in which we can deal with this situation. Our first solution is where a payment token allows a node to forward and receive packets through a relay node for a specified amount of time. This method could work well for voice telephony type applications. Alternatively if there is a one-to-one mapping of request and response packets, then we could assume that each node on the return path would forward one packet in the reverse direction. However in most situations, a single request may result in a number of packets being sent back as part of the response. To address this problem we could identify the nature of the request during the call setup phase. Each of the nodes the relay path would then send back their charge for packet forwarding based on the characteristics of the call.

\section{E. Redeeming Tokens}

Periodically a node will contact the broker and deposit payment tokens that it has collected for packet forwarding services that it provided to other nodes in the network. In Fig. 8 node L sends the highest hash value in the chain P5 that was spent at it by node A. The user's smart card module sends the hash value $\mathrm{P} 5_{20}$ along with the corresponding broker signed endorsement all encrypted with the public key of the broker. In addition the smart card digitally signs the message with the private key of node $\mathrm{L}$. The broker verifies the contents of the message, credits the account of node $\mathrm{L}$ and issues a receipt for 


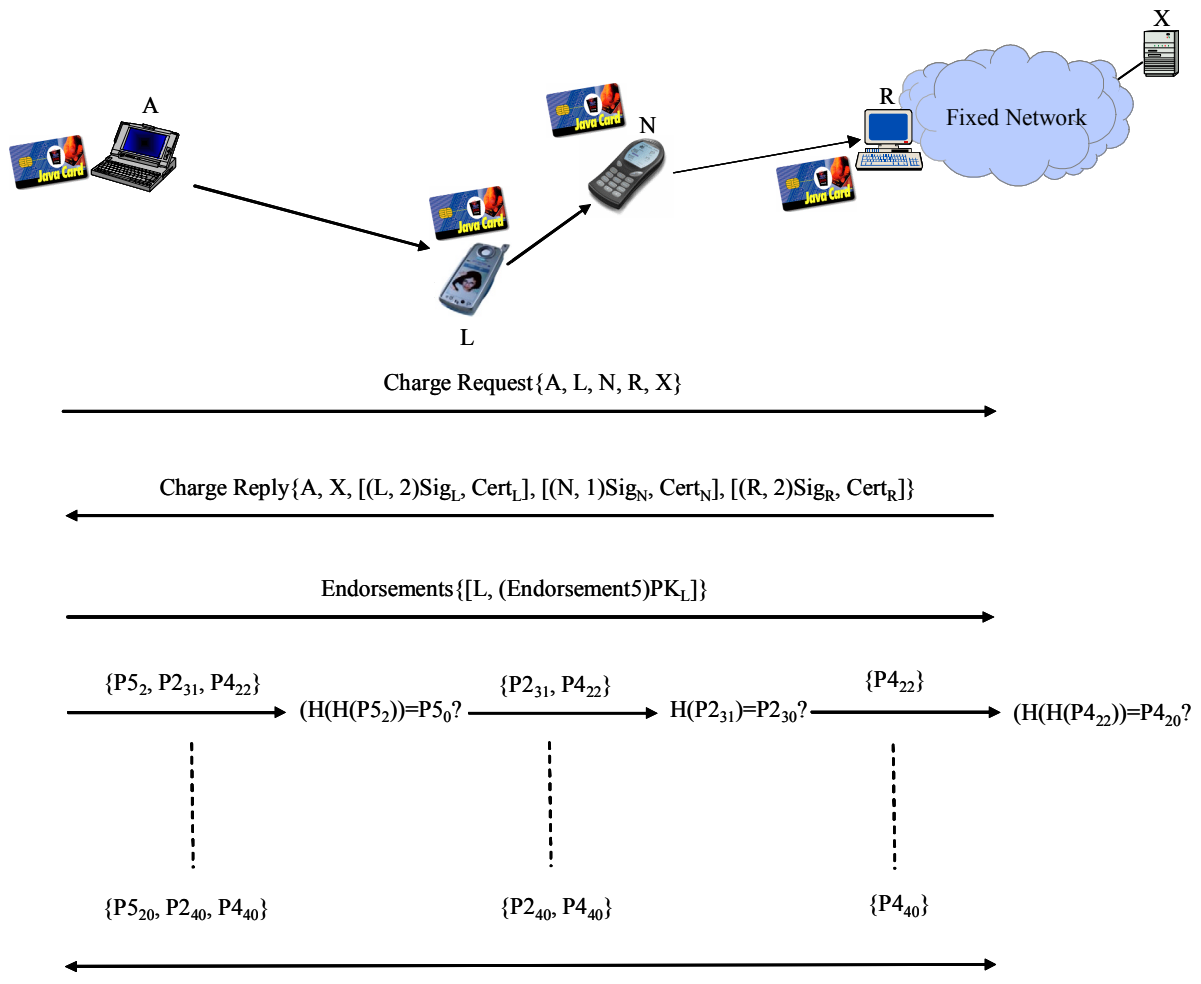

Distribute new set of endorsements to keep session alive

Figure 7. Payment of nodes on new path.

the same. At a later date the broker will also reimburse node A for the remaining 20 unspent hash values of the chain P5. The broker can automatically do this once the appropriate chains have expired.

\section{F. Broker Clearing}

Our discussions till now have focused on the use of a single broker in the system. A user usually contacts a local broker and opens an account with him. He receives a smart card module that is pre-loaded with the required root certificates. However the design of the payment protocol is such that there can be multiple brokers in the system, and individual users can be associated with any one of them. This allows for a more distributed system and avoids a central point of dependency in the system. Now when a user arrives in a new network, he contacts a local broker and purchases payment chains from him. In addition, he gets a copy of the local root certificates that he may require for verifying digital signatures generated by nodes within the new network. Contacting the broker using a secure terminal e.g. an ATM can be used to perform the initial priming operation. The brokers in the system can have accounting relationships and transfer funds between each other to settle user accounts.

\section{G. Discussion}

Our solution provides an efficient means of allowing realtime payment to multiple nodes in the relay path between the source and destination. A micropayment scheme using hash functions and off-line verification allows the solution to be efficient and scalable. For efficient storage of multiple hash chains on mobile devices we make use of unbalanced one-way binary trees. To aid performance asymmetric key algorithms are only used at call setup time and during subsequent endorsement distribution. In [10] the author has carried out a comprehensive study of micropayment performance. He has made efficiency comparisons of various hash algorithms versus symmetric and asymmetric key algorithms. One observation is with regards to the number of operations performed per second. It was noted that one could perform five times as many MD5 hash computations as DES encryption operations and over five thousand times as many RSA signature generations. In general the author observed that hashing is an order magnitude faster than symmetric encryption, three orders of magnitude faster than signature verification and four orders of magnitude faster than signature generation.

The payment scheme has been designed to be independent of the underlying routing protocol. This is essential as many types of routing algorithms have been defined and depending upon the type of network or environment one or more of them may be used. We have eliminated the use of long-lived payment contracts, which bind all the nodes in the payment path, as this model does not fit well in an ad hoc network scenario. Instead we securely obtain charging information from each node in the relay path and assemble a total charge for forwarding a packet through the network. Without access to an on-line TTP the only other solution is to make use of secure tamper resistant hardware modules. However we do not place total trust on the hardware modules contained within each node. If the tamper resistance of one of these devices is compromised our system will suffer from a limited amount of fraud. We assume that as these devices become more prevalent and powerful, that compromise of their security features will 
Receipt $\left\{\mathrm{L}, \mathrm{P} 5_{20}\right.$, Amount $\}$ Sig $_{\text {Broker }}$

Broker verifies hashes and credits users account

Figure 8. Redeeming payment hashes.

become an extremely difficult task. Also the expiry date associated with each chain of a UOBT prevents unlimited use of the chains in the system.

Hash chains are of a finite length and there is a possibility that a node may run out of hash values during a session. In the case of a UOBT if there are unused sub-chains, then the user can switch to the next chain by sending a new set of endorsements. If there were no further sub-chains available, then this would result in the dropping of a connection. This would be particularly true for real-time communications such as voice telephony or video conferencing, where the source may transmit a large number of datagrams during a session. However this applies to all payment protocols that have a fixed amount in the user's purse. Alternatively at call setup time a value which corresponds to the number of packets that an intermediate node will allow, before expecting the next hash value in the chain to be released by the source can be negotiated. For example each hash value could allow a source to transmit a hundred packets. This could be implemented simply as a counter at each node.

Each time we have a change in the topology in the ad hoc network, we need to distribute broker endorsements to each of the new nodes in the path. The amount of wastage in the system is dependent on two factors, namely node mobility and chain lengths. If there is high mobility in the network then we should make use small chains e.g. chains from a 10x10 UOBT. On the other hand if the routes in the network were relatively stable then it would make sense to use chains of longer lengths. One possibility is for a node to purchase UOBT's of different lengths from a broker. Since each hash value maybe worth a cent or less then the total value of the tree will not amount to a large sum. Depending on the network circumstances we could then make use chains from the appropriate tree.

\section{CONCLUSION}

With emergence of more mainstream type applications for ad hoc networks, there is need to move away from the notion of closed user groups, and the assumption that all nodes in an ad hoc network will cooperate with each other for benefit of the network as a whole. Current proposals for stimulating cooperation in ad hoc networks were examined and a number of drawbacks were identified. The authors concluded that a real world payment system could provide a good solution to resolving the problem of reliable forwarding of packets in ad hoc networks. The desirable properties of a payment system were then drawn up and a solution, which would achieve those goals, was proposed.

In this paper we have presented a method for compensating nodes for packet forwarding in an ad hoc network. Our proposed architecture allows routers to charge per-packet and adapts to routing changes. A node receives one or more routes to a destination along with a secure and verifiable charge for packet forwarding by each intermediate router. Based on this information the node can choose a suitable route and distribute endorsement values securely to each router in the path. The routers are able to verify the same and can immediately start accepting payment tokens for packet forwarding. Changes in the route between the source and destination do not require the source to contact a third party, such as a bank or broker to obtain payment tokens for the routers in the new path. It is able to immediately use additional chains to pay the new nodes in the routing path. We believe that such a system can lead to new mobile virtual network operators (MVNO) that hold promise for ubiquitous wireless voice and data network services.

\section{REFERENCES}

[1] L. Blazevic, L. Buttyán, S. Capkun, S. Giordano, J.-P. Hubaux and J.-Y. Le Boudec, "Self-Organization in Mobile Ad Hoc Networks: The Approach of Terminodes", IEEE Communications Magazine, June 2001.

[2] L. Buttyán and J.-P. Hubaux, "Nuglets: A Virtual Currency to Stimulate Cooperation in Self-Organized Mobile Ad Hoc Networks", Tech Report DSC/2001/001, Swiss Federal Institute of Technology, Lausanne, 2001.

[3] R. Rivest and A. Shamir, "PayWord and MicroMint: Two Simple Micropayment Schemes", Security Protocols, LNCS 1189, M. Lomas, Ed., Springer-Verlag, 1997, pp. 69-87, http://theory.lcs.mit.edu/ rivest

[4] R. Hauser, M. Steiner, and M. Waidner, "Micro-payments based on iKP", in Proc. of the 14th Worldwide Congress on Computer and Communications Security Protection, Paris, 1996, pp.67-82, http://www.zurich.ibm.com

[5] W3C Micropayments Working Group, http://www.w3.org/ECommerce/Micropayments/

[6] D. O'Mahony, M. Peirce and H. Tewari, Electronic Payment Systems for E-Commerce, $2^{\text {nd }}$ Ed., Artech House Publishers, Boston/London, 2001.

[7] L. Lamport, "Password Authentication with Insecure Communication", Communications of the ACM, vol. 24, no. 11, Nov. 1981, pp. 770-72.

[8] S. Yen, L. Ho and C. Huang, "Internet Micropayment Based on Unbalanced One-way Binary Tree", Proc. CrypTEC'99, Hong Kong, July 1999, pp.155-62.

[9] M. Peirce and D. O'Mahony, "Flexible Real-Time Payment Methods for Mobile Communications", IEEE Personal Communications, vol. 6, no. 6, Dec. 1999, pp. 44-55.

[10] M. Peirce, "Multi-party Micropayments for Mobile Communications", PhD Thesis, Trinity College Dublin, Ireland, Oct. 2000. 